\title{
COVID-19 Coagulopathy with Superior Mesenteric Vein Thrombosis Complicated by an Ischaemic Bowel
}

\author{
Bingwen Eugene Fan ${ }^{1,2,3,4}$ Cheng Chieh Ray Chang \\ ${ }^{1}$ Department of Haematology, Tan Tock Seng Hospital, Singapore \\ 2 Department of Laboratory Medicine, Khoo Teck Puat Hospital, \\ Singapore \\ ${ }^{3}$ Lee Kong Chian School of Medicine, Singapore \\ ${ }^{4}$ Yong Loo Lin School of Medicine, Singapore \\ ${ }^{5}$ Department of Pathology, Tan Tock Seng Hospital, Singapore \\ ${ }^{6}$ Department of Laboratory Medicine, National University Health \\ System, Singapore
}

Hämostaseologie 2020;40:592-593.

Address for correspondence Bingwen Eugene Fan, MBBS, MRCP, MMED, Department of Haematology, Tan Tock Seng Hospital, 11 Jalan Tan Tock Seng, Singapore 308433

(e-mail: Bingwen_Eugene_Fan@ttsh.com.sg).

\section{Abstract \\ Keywords \\ - abdominal pain \\ - COVID-19 \\ - hypercoagulability \\ - anticoagulation \\ - thrombosis}

COVID-19 coagulopathy is a hypercoagulable state which predisposes to venous, arterial and small vessel thrombosis. We describe a patient with COVID-19 who developed an acute superior mesenteric vein thrombosis with acute intestinal obstruction despite adequate anticoagulation.

\section{Introduction}

COVID-19 coagulopathy is a hypercoagulable state which predisposes to venous, arterial and small vessel thrombosis. ${ }^{1}$
We describe a patient with COVID-19 who developed an acute superior mesenteric vein (SMV) thrombosis with acute intestinal obstruction despite adequate anticoagulation.
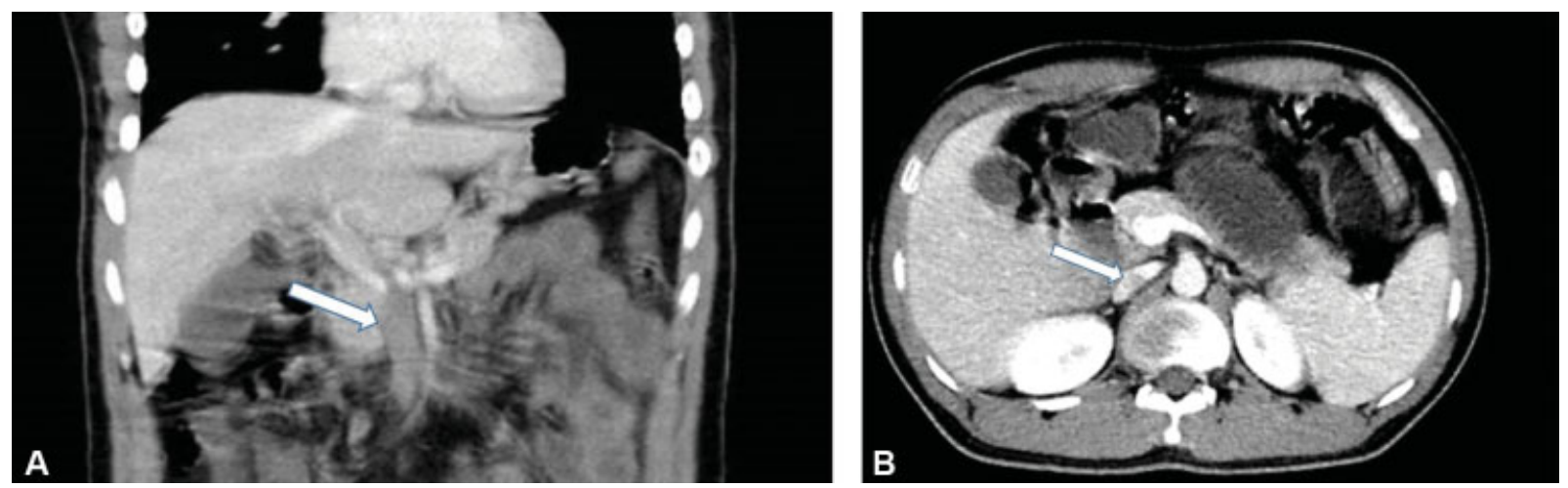

Fig. 1 (A) Superior mesenteric vein (SMV) thrombosis (filling defect seen highlighted by white arrow). (B) Dilated bowel loops extending from the duodenum to the jejunum. Interval improvement in the extent of previously noted SMV thrombosis, with interval reduction in calibre of the SMV and increased opacification. Filling defect seen in the SMV (highlighted by white arrow).

These 2 senior authors contributed equally.

received

July 15,2020

accepted

August 3, 2020 (c) 2020 Georg Thieme Verlag KG Stuttgart · New York
DOI https://doi.org/ 10.1055/a-1232-7446. ISSN 0720-9355. 

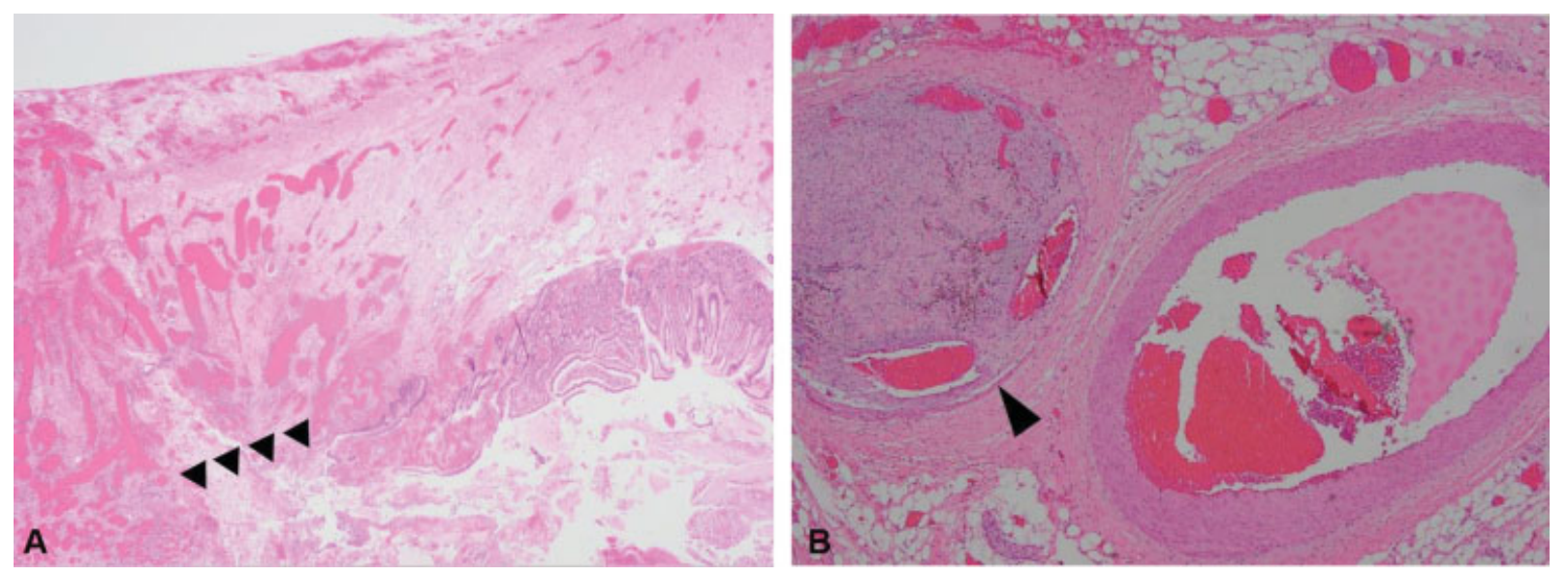

Fig. 2 (A) Histology of small bowel showing ulceration (black arrows), transmural congestion and haemorrhage (haematoxylin and eosin [H\&E] $\times 20)$. (B) Histology showing an organizing thrombus in a mesenteric vein (left vessel, black arrow) $(\mathrm{H} \& \mathrm{E} \times 40)$. A small mesenteric artery is seen on the right.

\section{Case Description}

A 30-year-old man presented to our institution on June 2020 with central abdominal pain and bilious vomiting for 2 days. He was previously diagnosed with COVID-19 and SMV thrombosis on May 2020 at another public hospital. CT of the abdomen and pelvis (CTAP) showed SMV thrombosis ( - Fig. 1A), with bilateral basal pneumonia. Nasopharyngeal swab sent for severe acute respiratory syndrome coronavirus 2 polymerase chain reaction was positive. Prothrombin time (PT) and activated partial thromboplastin time (aPTT) were normal, but lupus anticoagulant was positive with a mildly raised fibrinogen of $4.65 \mathrm{~g} / \mathrm{L}$ and markedly raised D-dimer of $>20.0 \mu \mathrm{g} / \mathrm{mL}$, consistent with COVID-19 coagulopathy. Twice-daily enoxaparin at $1 \mathrm{mg} / \mathrm{kg}$ was initiated and planned for 3 months. He was discharged well after a 17-day hospitalization. On review by our team, he claimed compliance to his anticoagulation. An urgent CTAP ( - Fig. 1B) showed small intestine obstruction. An exploratory laparotomy with resection of the affected small bowel loop was performed, with histology revealing ulceration, transmural congestion, haemorrhage ( $-\mathrm{Fig} .2 \mathrm{~A}$ ) and organizing thrombosis in mesenteric veins ( - Fig. $2 B$ ). Anti-cardiolipin IgG and IgM as well as anti-B2 glycoprotein, paroxysmal nocturnal haemoglobinuria (PNH) and molecular testing for myeloproliferative neoplasms (MPN) were all negative. Anticoagulation was resumed and the patient was discharged well.

\section{Conclusion}

This is a rare presentation of COVID-19-associated SMV thrombosis resulting in ischaemic bowel, with a lupus anticoagulant being frequently detected in COVID-19 patients as well. ${ }^{2}$ Clinicians encountering splanchnic vein thrombosis should be aware that this is an unusual site of thrombosis. ${ }^{3}$ In patients without obvious causes like liver cirrhosis, pancre- atitis, advanced malignancy, abdominal infection and postabdominal surgery, the exclusion of MPN, PNH, antiphospholipid syndrome and COVID-19 should be strongly considered. Given the increased incidence of COVID-19associated thromboembolic events ${ }^{4}$ and the associated morbidity and mortality, ${ }^{5}$ thromboprophylaxis should be strongly considered in hospitalized COVID-19 patients.

\section{Disclosures}

There was no conflict of interest declared for all authors and no funding was required. Written consent for publication was obtained from the patient.

\section{Conflict of Interest}

The authors declare, that they have no conflict of interest.

\section{Acknowledgements}

The COVID-19 Clotting and Bleeding Team at the National Centre for Infectious Diseases, Singapore, greatly appreciate the efforts of our fellow health care workers and the support of their families during this pandemic.

\section{References}

1 Becker RC. COVID-19 update: COVID-19-associated coagulopathy. J Thromb Thrombolysis 2020;50(01):54-67

2 Harzallah I, Debliquis A, Drénou B. Lupus anticoagulant is frequent in patients with Covid-19.J Thromb Haemost 2020;18(08):2064-2065

3 Tait C, Baglin T, Watson H, et al; British Committee for Standards in Haematology. Guidelines on the investigation and management of venous thrombosis at unusual sites. $\mathrm{Br} J$ Haematol 2012; 159(01):28-38

4 Klok FA, Kruip MJHA, van der Meer NJM, et al. . Incidence of thrombotic complications in critically ill ICU patients with COVID-19. Thromb Res 2020;191:145-147

5 Tang N, Li D, Wang X, Sun Z. Abnormal coagulation parameters are associated with poor prognosis in patients with novel coronavirus pneumonia. J Thromb Haemost 2020;18(04):844-847 